\title{
Star-based a Posteriori Error Estimator for Convection Diffusion Problems
}

\author{
B. Achchab ${ }^{1}$, A. Agouzal ${ }^{2, *}$, N. Debit ${ }^{2}$, K. Bouihat ${ }^{1}$ \\ ${ }^{1}$ Universite' Hassan $1^{e r}$, LM ${ }^{2}$ CE et LAMSAD, FSEJSS et ESTB, B.P 218 Berrechid, Morocco \\ ${ }^{2}$ Université de Lyon; CNRS ; Université Lyon 1 ; Institut Camille Jordan, \\ Bâtiment Jean Braconnier 21, Avenue Claude Bernard 69622 Villeurbanne cedex,France \\ *Corresponding Author: agouzal@univ-lyon1.fr
}

Copyright (C)2014 Horizon Research Publishing All rights reserved.

\begin{abstract}
In this paper, we derive an a posteriori error estimator, for nonconforming finite element approximation of convection-diffusion equation. The a posteriori error estimator is based on the local problems on stars. Finally, we prove the reliability and the efficiency of the estimator without saturation assumption nor comparison with residual estimator
\end{abstract}

Keywords A posteriori error estimator, nonconforming finite elements method, convection diffusion equations

AMS subject classification: 65D05, 65D15, 65N50

\section{Introduction}

A posteriori error estimators provide the basis for adaptive mesh refinement and quantitative error control $[1,8,4,5,12,19,14,10]$. One of the most successful estimators was proposed by Bank and Weiser and extended by many authors $[2,3,7,13,16,20,21]$, it is based on the solution of local Neumann problems on elements, which seem to allow for cancelation and thus lead to better results than the residual estimators. The classical proof of equivalence with the energy error require the saturation assumption: this says that the solution can be approximated asymptotically better with quadratic than with linear finite elements. The saturation assumption is shown to be superfluous by Nochetto in [17]. However, removing this assumption requires comparison with residual estimators. More recently, a new a posteriori error estimators on stars was proposed in [15], and the proof of the equivalence with energy error it applies directly without reference to residual estimators.

In this paper, we extended the results of [15] to the case of nonconforming finite elements and the convection diffusion case. A new a posteriori error estimator is introduced based on the solution of a small discrete problem in stars. We prove the reliability and the efficiency of the estimator without saturation assumption nor comparison with residual estimator. We consider the simpler case of nonconforming approximations for convection diffusion problem, and we introduced a technique which allowed us to define a new a posteriori error estimator which are equivalent to the energy error.

\section{Setting the problem}

We consider here the convection-diffusion problem:

$(P) \quad\left\{\begin{array}{c}-\varepsilon \Delta u+\beta . \nabla u=f \quad \text { in } \Omega, \\ u=0 \quad \text { on } \Gamma=\partial \Omega\end{array}\right.$

In the following we assume that $\Omega \in \mathbf{R}^{2}$ a simply connected polygon domain, $0<\varepsilon<<1, \beta \in\left(W^{1, \infty}(\Omega)\right)^{2}$, such that $-\frac{1}{2} \operatorname{div} \beta \geq a>0$ and $f \in L^{2}(\Omega)$. Let $\mathcal{T}_{h}$ be a family of conforming shape-regular triangulation of $\Omega$ by triangular, we denoted by $E_{I}$ the set of interior edges and by $E_{f}$ the set of all edges included in $\Gamma$. Let $V_{h}$ be the 
lowest order non-conforming Crouzeix-Raviart finite element space defined by:

$$
\begin{aligned}
V_{h}= & \left\{v_{h} \in L^{2}(\Omega) ; \forall T \in \mathcal{T}_{h}, v_{h \mid T} \in P_{1}(T),\right. \\
& \forall E \in E_{I}, \int_{E}\left[v_{h}\right]_{E} d \sigma=0 \text { and } \forall E \in E_{f}, \\
& \left.\int_{E} v_{h} d \sigma=0\right\} .
\end{aligned}
$$

where $[.]_{E}$ denoted the jump of the function across $E$. For each $T \in \mathcal{T}_{h}$, we denote by $P_{k}(T)$ the polynomial space of degree less than or equal to $k$.

For all $T \in \mathcal{T}_{h}$, we define $\partial T^{-}$such the part of the frontier of $T$ such that $\beta . n_{T}<0$ where $n_{T}$ stands for the unit outward normal vector to $T$ on $\partial T$.

In the sequel, we consider $u_{h}^{N C} \in V_{h}$ be a solution of the stabilized nonconforming approximation problem:

$$
\left(P_{h}\right)^{N C}\left\{\begin{array}{l}
\forall v_{h} \in V_{h}, \\
\sum_{T \in \mathcal{T}_{h}} \int_{T}\left[\varepsilon \nabla u_{h}^{N C} \cdot \nabla v_{h}+\beta \cdot \nabla u_{h}^{N C} v_{h}\right] d x \\
+\frac{1}{2} \sum_{T \in \mathcal{T}_{h}} \int_{\partial T^{-}} \beta \cdot n\left[u_{h}^{N C}\right] v_{h} d \sigma=\int_{\Omega} f v_{h} d x
\end{array}\right.
$$

\subsubsection{The a posteriori error estimator}

For the a posteriori error analysis of the considered approximation, we need to define some local spaces and problems. We denoted by $\left\{x_{i}\right\}_{i \in \mathcal{N}}$ the set of all nodes of the triangulation $\mathcal{T}_{h}$. For each $i \in \mathcal{N}, \phi_{i}$ denoted the canonical continuous piecewise linear basis function corresponding to $x_{i}$. The star $\omega_{i}$ is the interior relative to $\Omega$ of the support of $\phi_{i}$, and $h_{i}$ is the maximal size of the elements forming $\omega_{i}$. Finally, $\Gamma_{i}$ will denote the union of the sides touching $x_{i}$ that are contained in $\Omega$, and $\bar{\Gamma}_{i}$ will denote the union of the sides touching $x_{i}$ that are contained in $\bar{\Omega}$.

For each star $\omega_{i}, i \in \mathcal{N}$, if $x_{i}$ we introduce the space $V\left(\omega_{i}\right)$ defined by

$$
V\left(\omega_{i}\right)=\left\{v \in H_{l o c}^{1}\left(\omega_{i}\right): \int_{\omega_{i}} v \phi_{i} d x=0\right\},
$$

if $x_{i}$ is an interior node, and

$$
V\left(\omega_{i}\right)=\left\{v \in H_{l o c}^{1}\left(\omega_{i}\right): v=0 \text { on } \partial \omega_{i} \cap \Gamma\right\},
$$

if $x_{i}$ is a boundary node.

We have the following result of $([15])$

Proposition 1 There exists a constant $C$, only depending on the minimum angle of the triangulation but independent of the star being considered, such that:

$$
\forall v \in V\left(\omega_{i}\right) \quad\|v\|_{0, \omega_{i}} \leq C h_{i}\left(\int_{\omega_{i}}|\nabla v|^{2} \phi_{i} d x\right)^{1 / 2} .
$$

We define the finite dimensional local spaces $\mathcal{P}_{0}^{2}\left(\omega_{i}\right)$ as follows,

Definition 2 For $i \in \mathcal{N}$, let $\mathcal{P}^{2}\left(\omega_{i}\right)$ denote the space of continuous piecewise quadratic functions on star $\omega_{i}$ that vanish on $\partial \omega_{i}$. The spaces $\mathcal{P}_{0}^{2}\left(\omega_{i}\right)$ is defined by $\mathcal{P}_{0}^{2}\left(\omega_{i}\right)=\mathcal{P}^{2}\left(\omega_{i}\right) \cap V\left(\omega_{i}\right)$.

In the following we consider the energy norm:

$$
\|u\|_{\varepsilon, \omega_{i}}^{2}=\varepsilon\|\nabla u\|_{0, \omega_{i}}^{2}+\|u\|_{0, \omega_{i}}^{2} .
$$

Let $u_{h}^{N C} \in V_{h}$ be fixed and we denoted by $\nabla_{h} u_{h}$ the vector belonging to $\left(L^{2}(\Omega)\right)^{2}$ defined by

$$
\forall T \in \mathcal{T}_{h} ; \quad \nabla_{h} v_{h}=\nabla v_{h} \quad \text { on } T .
$$


For each $i \in \mathcal{N}$, we consider the local problems :

$$
\left(P_{1}\right)_{i} \quad\left\{\begin{array}{l}
\text { Find } \eta_{i} \in \mathcal{P}_{0}^{2}\left(\omega_{i}\right) \text { such that } \forall \mu_{i} \in \mathcal{P}_{0}^{2}\left(\omega_{i}\right) \\
\int_{\omega_{i}}\left(\varepsilon \nabla \eta_{i} \cdot \nabla \mu_{i}\right) \phi_{i} d x=\int_{\omega_{i}}\left(\varepsilon \nabla h u_{h}^{N C} \cdot \nabla \mu_{i}\right) \phi_{i} d x \\
+\int_{\omega_{i}}\left(\beta \cdot \nabla_{h} u_{h}^{N C} \mu_{i}\right) \phi_{i} d x \\
+\frac{1}{2} \sum_{T \in \mathcal{T}_{h}} \int_{\partial T^{-}} \beta \cdot n\left[u_{h}^{N C}\right] \mu_{i} \phi_{i} d \sigma-\int_{\omega_{i}} f \mu_{i} \phi_{i} d x
\end{array}\right.
$$

and

$$
\left(P_{2}\right)_{i} \quad\left\{\begin{array}{l}
\text { Find } \alpha_{i} \in \mathcal{P}_{0}^{2}\left(\omega_{i}\right) \text { such that } \forall \mu_{i} \in \mathcal{P}_{0}^{2}\left(\omega_{i}\right) \\
\int_{\omega_{i}}\left(\varepsilon \nabla \alpha_{i} \cdot \nabla \mu_{i}\right) \phi_{i} d x=\int_{\omega_{i}} \varepsilon \nabla_{h} u_{h}^{N C} \cdot \operatorname{Curl}\left(\mu_{i} \phi_{i}\right) d x .
\end{array}\right.
$$

Using Lax-Milgram theorem, we prove that the discrete problems have unique solution. The problems $\left(P_{1}\right)_{i}$ estimate the approximation error, but the problems $\left(P_{2}\right)_{i}$ estimate the consistency error of the used method.

Finally we set:

$$
\forall i \in \mathcal{N}, \quad E_{1, i}^{2}\left(u_{h}^{N C}\right)=\int_{\omega_{i}} \varepsilon\left|\nabla \eta_{i}\right|^{2} \phi_{i} d x
$$

and

$$
\forall i \in \mathcal{N}, \quad E_{2, i}^{2}\left(u_{h}^{N C}\right)=\int_{\omega_{i}} \varepsilon\left|\nabla \alpha_{i}\right|^{2} \phi_{i} d x
$$

\subsubsection{Upper bound of the error}

In this section we prove the one of main results of this paper. First, we prove the upper bound of the error without oscillation. As in $([6],[9],[11])$. Recall that [18]:

Lemma 3 (Discrete Poincar and Friedrichs inequalities).

There is a positives constants $C$ depending only on the minimum angle of $\mathcal{T}_{h}$ the $\Omega$ such that:

$$
\forall v \in H_{0}^{1}(\Omega)+V_{h}, \quad\|v\|_{0, \Omega}^{2} \leq C\left(\sum_{T \in \mathcal{T}_{h}}\|\nabla v\|_{0, \Omega}^{2}\right) .
$$

We have the following global upper bond of the error:

Theorem 4 Let $u_{h}^{N C} \in V_{h}$ such that $\left(P_{h}\right)^{N C}$ holds. There is a positive constant $C_{1}$ depending only on the minimum angle of $\mathcal{T}_{h}$ such that

$$
\begin{aligned}
\left\|u-u_{h}^{N C}\right\|_{\varepsilon, \Omega} & \leq C_{1}\left[\left(\sum_{i \in \mathcal{N}}\left(E_{1, i}^{2}\left(u_{h}^{N C}\right)+E_{2, i}^{2}\left(u_{h}^{N C}\right)\right)\right)^{\frac{1}{2}}\right. \\
& +\|\beta\|_{1, \infty} h_{i}\left(\sum_{i \in \mathcal{N}} E_{2, i}^{2}\left(u_{h}^{N C}\right)\right)^{\frac{1}{2}}, \\
& +\sum_{i \in \mathcal{N}}\left(h_{i} \sum_{T \subset \omega_{i}}\left\|\left[u_{h}^{N C}\right]\right\|_{L^{2}(\partial T)}\right. \\
& \left.+\left(\|\beta\|_{0, \infty}+\|\beta\|_{H\left(\operatorname{div}, \omega_{i}\right)}\right) h_{i}\left\|u_{h}^{N C}\right\|_{0, \omega_{i}}\right) \\
& +\operatorname{osc}(f)] .
\end{aligned}
$$

where osc $(f)$ is the data oscillations defined by: osc $(f)=\left(\sum_{i \in \mathcal{N}} \alpha_{i}^{2}\left\|\left(f-f_{i}\right) \phi_{i}^{\frac{1}{2}}\right\|_{0, \omega_{i}}\right)^{\frac{1}{2}}$.

where $f_{i}=\frac{\int_{\omega_{i}} f \phi_{i} d x}{\int_{\omega_{i}} \phi_{i} d x} \quad$ and $\quad \alpha_{i}=\min \left(1, \frac{h_{i}}{\sqrt{\varepsilon}}\right)$.

Proof. Remark that using Helmholtz-decomposition, we have

$$
\nabla_{h} u_{h}^{N C}-\nabla u=\nabla w+\operatorname{Curl} \zeta
$$

with $w \in H_{0}^{1}(\Omega), \zeta \in H^{1}(\Omega)$ and $\int_{\Omega} \nabla w \cdot \operatorname{Curl} \zeta d x=0$.

Let us remark also that the orthogonality implies the following error decomposition :

$$
\varepsilon\left\|\nabla_{h} u_{h}^{N C}-\nabla u\right\|_{0, \Omega}^{2}=\varepsilon\|\nabla w\|_{0, \Omega}^{2}+\varepsilon\|\operatorname{Curl} \zeta\|_{0, \Omega}^{2} .
$$

and the following equalities :

$$
\varepsilon\|\nabla w\|_{\Omega}^{2}=\int_{\Omega} \varepsilon\left(\nabla_{h} u_{h}^{N C}-\nabla u\right) \cdot \nabla w d x .
$$


and

$$
\varepsilon\|\operatorname{Curl} \zeta\|_{0, \Omega}^{2}=\int_{\Omega} \varepsilon\left(\nabla_{h} u_{h}^{N C}-\nabla u\right) \cdot \operatorname{Curl} \zeta d x .
$$

The estimates of expressions in (5) and (6) will be established respectively in the Lemmas 7 and 8 . As a main tool we use the following Lemmas.

Lemma 5 For each node $i \in \mathcal{N}$ there exists an unique operator

$\Pi_{i}: V\left(\omega_{i}\right) \longrightarrow \mathcal{P}_{0}^{2}\left(\omega_{i}\right)$, such that for any $v \in V\left(\omega_{i}\right)$ the following conditions hold :

1. For all edge $E \subset \Gamma_{i}, \int_{E}\left(v-\Pi_{i} v\right) \phi_{i} d \sigma=0$.

2. $\int_{\omega_{i}}\left(v-\Pi_{i} v\right) \phi_{i} d x=0$

3. $\left.\varepsilon\left(\int_{\omega_{i}}\left|\nabla \Pi_{i} v\right|^{2} \phi_{i}\right)^{\frac{1}{2}}+\left(\int_{\omega_{i}}\left|\Pi_{i} v\right|^{2} \phi_{i}\right)^{\frac{1}{2}} \leq C\left[\varepsilon\left(\int_{\omega_{i}}|\nabla v|^{2} \phi_{i}\right)^{\frac{1}{2}}+\int_{\omega_{i}}|v|^{2} \phi_{i}\right)^{\frac{1}{2}}\right]$,

4. $\left(\sum_{i \in \mathcal{N}} \alpha_{i}^{-2}\left\|\left(v-\Pi_{i}(v)\right)\left(\phi_{i}\right)^{\frac{1}{2}}\right\|_{0, \omega_{i}}^{2}\right)^{\frac{1}{2}} \leq C\|v\|_{\varepsilon, \Omega}$

where the constant $C$ depends only on the minimum angles of $\mathcal{T}_{h}$.

The Lemma 5, is an adaptation of arguments given in [15], and so the proof will be skipped.

Lemma 6 For each node $i \in \mathcal{N}, \Pi_{i}$ defined in the 5, functions $v \in V\left(\omega_{i}\right), \zeta \in V\left(\omega_{i}\right)$ and $u_{h}^{N C} \in V_{h}$. We have

$$
\int_{\omega_{i}} \varepsilon \nabla_{h} u_{h}^{N C} \cdot \nabla\left(\left(\Pi_{i} v\right) \phi_{i}\right) d x=\int_{\omega_{i}} \varepsilon \nabla_{h} u_{h}^{N C} \cdot \nabla\left(v \phi_{i}\right) d x
$$

and

$$
\int_{\omega_{i}} \varepsilon \nabla_{h} u_{h}^{N C} \cdot \operatorname{Curl}\left(\left(\Pi_{i} \zeta\right) \phi_{i}\right) d x=\int_{\omega_{i}} \varepsilon \nabla_{h} u_{h}^{N C} \cdot \nabla\left(\zeta \phi_{i}\right) d x .
$$

Proof. If we denoted by $\left[\frac{\partial u_{h}^{N C}}{\partial n_{E}}\right] \in P_{0}(E)$ the jump of the normal derivative across the edge E. By Green formula and by lemma 5 , we have

$$
\begin{aligned}
& \int_{\omega_{i}} \varepsilon \nabla_{h} u_{h}^{N C} \cdot \nabla\left(\left(\Pi_{i} v\right) \phi_{i}\right) d x \\
= & \sum_{E \subset \omega_{i}} \int_{E} \varepsilon\left[\frac{\partial u_{h}^{N C}}{\partial n_{E}}\right]\left(\Pi_{i} v\right) \phi_{i} d x \\
= & \sum_{E \subset \omega_{i}} \int_{E} \varepsilon\left[\frac{\partial u_{h}^{N C}}{\partial n_{E}}\right] v \phi_{i} d x=\int_{\omega_{i}} \varepsilon \nabla_{h} u_{h}^{N C} \cdot \nabla\left(v \phi_{i}\right) d x .
\end{aligned}
$$

To prove the second equality, if we denoted by $\left[\frac{\partial u_{h}^{N C}}{\partial \tau_{E}}\right] \in P_{0}(E)$ the jump of the tangential derivative across the edge E. We have by Green formula and lemma 5

$$
\begin{gathered}
\int_{\omega_{i}} \varepsilon \nabla_{h} u_{h}^{N C} \cdot \operatorname{Curl}\left(\left(\Pi_{i} \zeta\right) \phi_{i}\right) d x=\sum_{E \subset \omega_{i}} \int_{E} \varepsilon\left[\frac{\partial u_{h}^{N C}}{\partial \tau_{E}}\right]\left(\Pi_{i} \zeta\right) \phi_{i} d x \\
=\sum_{E \subset \omega_{i}} \int_{E} \varepsilon\left[\frac{\partial u_{h}^{N C}}{\partial \tau_{E}}\right] \zeta \phi_{i} d x=\int_{\omega_{i}} \varepsilon \nabla_{h} u_{h}^{N C} \cdot \operatorname{Curl}\left(\zeta \phi_{i}\right) d x .
\end{gathered}
$$

The following Lemma give an estimate of expression (5):

Lemma 7 For $w \in H_{0}^{1}(\Omega)$ defined in (4), there exists positives constants $C^{*}$, depending only on the minimum angle of $\mathcal{T}_{h}$ such that

$$
\begin{aligned}
\|w\|_{\varepsilon, \Omega} & \leq C^{*}\left[\left(\sum_{i \in \mathcal{N}}\left(E_{1, i}^{2}\left(u_{h}^{N C}\right)\right)\right)^{\frac{1}{2}}\right. \\
& +\|\beta\|_{1, \infty} h_{i}\left(\sum_{i \in \mathcal{N}} E_{2, i}^{2}\left(u_{h}^{N C}\right)\right)^{\frac{1}{2}}, \\
& +\sum_{i \in \mathcal{N}}\left(h_{i} \sum_{T \subset \omega_{i}}\left\|\left[u_{h}^{N C}\right]\right\|_{L^{2}(\partial T)}+\left(\|\beta\|_{0, \infty}\right.\right. \\
& \left.\left.+\|\beta\|_{H\left(\operatorname{div}, \omega_{i}\right)}\right) h_{i}\left\|u_{h}^{N C}\right\|_{0, \omega_{i}}\right), \\
& +\operatorname{osc}(f)] .
\end{aligned}
$$


Proof . For $w \in H_{0}^{1}(\Omega)$ defined in (4), we set $w_{h}=\sum_{i \in \mathcal{N}} w_{i} \phi_{i}$, where $w_{i}=\frac{\int_{\omega_{i}} w \phi_{i} d x}{\int_{\omega_{i}} \phi_{i} d x}$ for interior nodes, and $w_{i}=0$ otherwise.

By adapting standard arguments used in the analysis of finite element approximation, and by using the propriety $-\frac{1}{2} \operatorname{div} \beta \geq a>0$, wa have:

$$
\begin{aligned}
a\|w\|_{\varepsilon, \Omega}^{2} & \leq \int_{\Omega} \varepsilon \nabla w \cdot \nabla w \\
& +\int_{\Omega} \beta \cdot \nabla w w d x
\end{aligned}
$$

where: $\varepsilon\|\nabla w\|_{\Omega}^{2}=\int_{\Omega} \varepsilon\left(\nabla_{h} u_{h}^{N C}-\nabla u\right) . \nabla w d x \quad$ and $\int_{\Omega} \nabla w \cdot \operatorname{Curl} \zeta d x=0$

This gives,

$$
\begin{aligned}
\int_{\Omega} \varepsilon \nabla w \cdot \nabla w+\int_{\Omega} \beta \cdot \nabla w w= & \int_{\Omega} \varepsilon \nabla w \cdot\left(\nabla_{h} u_{h}^{N C}-\nabla u\right) d x \\
& +\int_{\Omega} \beta \cdot\left(\nabla_{h} u_{h}^{N C}-\nabla u\right) w \\
& -\int_{\Omega} \beta \cdot w \operatorname{Curl} \zeta d x \\
& -\int_{\Omega} \varepsilon \cdot \nabla w \cdot \operatorname{Curl} \zeta d x \\
= & \int_{\Omega} \varepsilon \nabla_{h} u_{h}^{N C} \cdot \nabla w d x \\
+ & \int_{\Omega} \beta \cdot \nabla_{h} u_{h}^{N C} w d x \\
& -\int_{\Omega} \varepsilon \nabla u \cdot \nabla w d x-\int_{\Omega} \beta . \nabla u w d x \\
& -\int_{\Omega} \beta \cdot w \operatorname{Curl} \zeta d x \\
& \int_{\Omega} \varepsilon \cdot \nabla w \cdot \operatorname{Curl} \zeta d x .
\end{aligned}
$$

Since $w_{h} \in V_{h} \cap H_{0}^{1}(\Omega), a\left(w, w_{h}\right)=0$, we have: $a(w, w)=a\left(w, w-w_{h}\right)$. Then

$$
\begin{aligned}
a\|w\|_{\varepsilon, \Omega}^{2} & \leq \int_{\Omega} \varepsilon \nabla_{h} u_{h}^{N C} \cdot \nabla\left(w-w_{h}\right) d x \\
& +\int_{\Omega} \beta \cdot \nabla_{h} u_{h}^{N C}\left(w-w_{h}\right) d x \\
& -\int_{\Omega} \varepsilon \nabla u \cdot \nabla\left(w-w_{h}\right) d x-\int_{\Omega} \beta \cdot \nabla u\left(w-w_{h}\right) d x \\
& -\int_{\Omega} \beta \cdot\left(w-w_{h}\right) \operatorname{Curl} \zeta d x .
\end{aligned}
$$

Stating that

$w-w_{h}=\sum_{i \in \mathcal{N}}\left(w-w_{i}\right) \phi_{i}$, and using $\sum_{i \in \mathcal{N}} \phi_{i}=1$ gives

$$
\begin{aligned}
a\|w\|_{\varepsilon, \Omega}^{2} & \leq \sum_{i \in \mathcal{N}}\left[\int_{\omega_{i}} \varepsilon \nabla_{h} u_{h}^{N C} \cdot \nabla\left(w-w_{i}\right) \phi_{i} d x\right. \\
& \left.+\int_{\omega_{i}} \beta \cdot \nabla_{h} u_{h}^{N C}\left(w-w_{i}\right) \phi_{i} d x\right] \\
& -\sum_{i \in \mathcal{N}}\left[\int_{\omega_{i}} f\left(w-w_{i}\right) \phi_{i} d x\right. \\
& \left.-\int_{\omega_{i}} \beta \cdot\left(w-w_{i}\right) \phi_{i} \operatorname{Curl} \zeta d x\right]
\end{aligned}
$$


Since $\left(w-w_{i}\right) \in V\left(\omega_{i}\right)$, adding and removing same quantities in the two last terms give:

$$
\begin{aligned}
& a\|w\|_{\varepsilon, \Omega}^{2} \\
& \leq \sum_{i \in \mathcal{N}}\left[\int_{\omega_{i}} \varepsilon \nabla_{h} u_{h}^{N C} \cdot \nabla \Pi_{i}\left(w-w_{i}\right) \phi_{i}\right) d x \\
& \left.+\int_{\omega_{i}} \beta \cdot \nabla_{h} u_{h}^{N C} \Pi_{i}\left(w-w_{i}\right) \phi_{i} d x\right] \\
& +\sum_{i \in \mathcal{N}}\left[\frac{1}{2} \sum_{T \subset \omega_{i}} \int_{\partial T^{-}}|\beta \cdot n|\left[u_{h}^{N C}\right]\left(\Pi_{i}\left(w-w_{i}\right)\right) \phi_{i} d \sigma\right] \\
& -\sum_{i \in \mathcal{N}}\left[\int_{\omega_{i}} f \Pi_{i}\left(w-w_{i}\right) \phi_{i} d x\right] \\
& -\sum_{i \in \mathcal{N}}\left[\frac{1}{2} \sum_{T \subset \omega_{i}} \int_{\partial T^{-}}|\beta . n|\left[u_{h}^{N C}\right]\left(\Pi_{i}\left(w-w_{i}\right)\right) \phi_{i} d \sigma\right] \\
& +\sum_{i \in \mathcal{N}}\left[\int_{\omega_{i}} \beta . \nabla_{h} u_{h}^{N C}\left(w-w_{i}-\Pi_{i}\left(w-w_{i}\right)\right) \phi_{i} d x\right] \\
& -\sum_{i \in \mathcal{N}}\left[\int_{\omega_{i}} f\left(w-w_{i}-\Pi_{i}\left(w-w_{i}\right)\right) \phi_{i} d x\right] \\
& +\left[\int_{\omega_{i}} \beta .\left(w-w_{i}\right) \phi_{i} \operatorname{Curl} \zeta d x\right] .
\end{aligned}
$$

Using the definition of local problems $\left(P_{1}\right)_{i}$,

$$
\begin{aligned}
& a\|w\|_{\varepsilon, \Omega}^{2} \\
& \left.\leq \sum_{i \in \mathcal{N}}\left[\int_{\omega_{i}} \varepsilon \nabla \eta_{i} \cdot \nabla \Pi_{i}\left(w-w_{i}\right) \phi_{i}\right) d x\right] \\
& -\sum_{i \in \mathcal{N}}\left[\int_{\omega_{i}} f\left(w-w_{i}-\Pi_{i}\left(w-w_{i}\right)\right) \phi_{i} d x\right] \\
& -\sum_{i \in \mathcal{N}}\left[\frac{1}{2} \sum_{T \subset \omega_{i}} \int_{\partial T^{-}}|\beta \cdot n|\left[u_{h}^{N C}\right]\left(\Pi_{i}\left(w-w_{i}\right)\right) \phi_{i} d \sigma\right] \\
& +\left[\sum_{i \in \mathcal{N}} \int_{\omega_{i}} \beta . \nabla_{h} u_{h}^{N C}\left(w-w_{i}-\Pi_{i}\left(w-w_{i}\right)\right) \phi_{i} d x\right] \\
& -\sum_{i \in \mathcal{N}}\left[\int_{\omega_{i}} \beta .\left(w-w_{i}\right) \phi_{i} \operatorname{Curl} \zeta d x\right] .
\end{aligned}
$$

We now process successively with each term of the right-hand side. On one hand, using Cauchy-Schwarz and item 3 of lemma 5 we have:

$$
\begin{aligned}
& \int_{\omega_{i}} \varepsilon \nabla \eta_{i} \cdot \nabla \Pi_{i}\left(w-w_{i}\right) \phi_{i} d x \\
& \leq\left(\sum_{i \in \mathcal{N}} \int_{\omega_{i}} \varepsilon\left|\nabla \eta_{i}\right|^{2} \phi_{i} d x\right)^{\frac{1}{2}}\left(\sum_{i \in \mathcal{N}} \int_{\omega_{i}} \varepsilon\left|\nabla \Pi_{i}\left(w-w_{i}\right)\right|^{2} \phi_{i} d x\right)^{\frac{1}{2}} \\
& \leq C\left(\sum_{i \in \mathcal{N}} E_{1, i}^{2}\left(u_{h}^{N C}\right)\right)^{\frac{1}{2}}\left(\sum_{i \in \mathcal{N}} \int_{\omega_{i}} \varepsilon\left|\nabla\left(w-w_{i}\right)\right|^{2} \phi_{i} d x\right)^{\frac{1}{2}}, \\
& \leq C\left(\sum_{i \in \mathcal{N}} E_{1, i}^{2}\left(u_{h}^{N C}\right)\right)^{\frac{1}{2}}\|w\|_{\varepsilon, \Omega} .
\end{aligned}
$$

On the other hand, since both of $\left(w-w_{i}\right)$ and $\Pi_{i}\left(w-w_{i}\right)$ belong to $V\left(w_{i}\right)$, using definition of $V\left(w_{i}\right)$ and coefficients $f_{i}$ give:

$$
\begin{aligned}
& \sum_{i \in \mathcal{N}}\left[\int_{\omega_{i}} f\left(w-w_{i}-\Pi_{i}\left(w-w_{i}\right)\right) \phi_{i} d x\right] \\
& =\sum_{i \in \mathcal{N}}\left[\int_{\omega_{i}}\left(f-f_{i}\right)\left(w-w_{i}-\Pi_{i}\left(w-w_{i}\right)\right) \phi_{i} d x\right]
\end{aligned}
$$


Using Cauchy-Schwarz, the proposition 1, the item 4 of lemma 5 and once more $\sum_{i \in \mathcal{N}} \phi_{i}=1$ we get

$$
\begin{aligned}
& \sum_{i \in \mathcal{N}}\left[\int_{\omega_{i}} f\left(w-w_{i}-\Pi_{i}\left(w-w_{i}\right)\right) \phi_{i} d x\right] \\
& \leq \operatorname{osc}(f)\left(\sum_{i \in \mathcal{N}} \alpha_{i}^{-2}\left\|\left(w-w_{i}-\Pi_{i}\left(w-w_{i}\right)\right)\left(\phi_{i}\right)^{\frac{1}{2}}\right\|_{0, \omega_{i}}^{2}\right)^{\frac{1}{2}} \\
& \leq C \operatorname{osc}(f)\|w\|_{\varepsilon, \Omega} .
\end{aligned}
$$

$C$ is a generic constant only depending on the minimum angle of triangulation.

We note:

$$
\begin{aligned}
A & =\sum_{i \in \mathcal{N}}\left[\int_{\omega_{i}} \beta \cdot \nabla_{h} u_{h}^{N C}\left(w-w_{i}-\Pi_{i}\left(w-w_{i}\right)\right) \phi_{i} d x\right] \\
& -\left[\frac{1}{2} \sum_{T \subset \omega_{i}} \int_{\partial T^{-}}|\beta . n|\left[u_{h}^{N C}\right]\left(\Pi_{i}\left(w-w_{i}\right)\right) \phi_{i} d \sigma\right]
\end{aligned}
$$

Using the Green formula we have:

$$
\begin{aligned}
A & =\sum_{i \in \mathcal{N}}\left[\int_{\omega_{i}} \beta . \nabla_{h} u_{h}^{N C}\left(w-w_{i}-\Pi_{i}\left(w-w_{i}\right)\right) \phi_{i} d x\right] \\
- & {\left[\frac{1}{2} \sum_{T \subset \omega_{i}} \int_{\partial T^{-}}|\beta \cdot n|\left[u_{h}^{N C}\right]\left(\Pi_{i}\left(w-w_{i}\right)\right) \phi_{i} d \sigma\right] } \\
= & \sum_{i \in \mathcal{N}}\left[\frac{1}{2} \sum_{T \in \mathcal{T}_{h}} \int_{\partial T^{-}}|\beta . n|\left[u_{h}^{N C}\right]\left(w-w_{i}-\Pi_{i}\left(w-w_{i}\right)\right) \phi_{i} d \sigma\right] \\
- & \sum_{i \in \mathcal{N}}\left[\int_{\omega_{i}} \beta . u_{h}^{N C} \nabla\left(w-w_{i}-\Pi_{i}\left(w-w_{i}\right)\right) \phi_{i} d x\right] \\
+ & \sum_{i \in \mathcal{N}}\left[\int_{\omega_{i}} \operatorname{div} \beta . u_{h}^{N C}\left(w-w_{i}-\Pi_{i}\left(w-w_{i}\right)\right) \phi_{i} d x\right] \\
- & {\left[\frac{1}{2} \sum_{T \subset \omega_{i}} \int_{\partial T^{-}}|\beta . n|\left[u_{h}^{N C}\right]\left(\Pi_{i}\left(w-w_{i}\right)\right) \phi_{i} d \sigma\right] . }
\end{aligned}
$$

Using Cauchy-Schwarz and the proposition 1 we have:

$$
\begin{aligned}
A & =\sum_{i \in \mathcal{N}}\left[\int_{\omega_{i}} \beta \cdot \nabla_{h} u_{h}^{N C}\left(w-w_{i}-\Pi_{i}\left(w-w_{i}\right)\right) \phi_{i} d x\right] \\
& -\left[\frac{1}{2} \sum_{T \subset \omega_{i}} \int_{\partial T^{-}}|\beta . n|\left[u_{h}^{N C}\right]\left(\Pi_{i}\left(w-w_{i}\right)\right) \phi_{i}\right] \\
& \leq C\left[\sum _ { i \in \mathcal { N } } \left(h_{i} \sum_{T \subset \omega_{i}}\left\|\left[u_{h}^{N C}\right]\right\|_{L^{2}(\partial T)}+\left(\|\beta\|_{0, \infty}\right.\right.\right. \\
& \left.\left.\left.+\|\beta\|_{H\left(\operatorname{div}, \omega_{i}\right)}\right) h_{i}\left\|u_{h}^{N C}\right\|_{0, \omega_{i}}\right)+\operatorname{osc}(f)\right] .
\end{aligned}
$$

Finally:

$$
\begin{aligned}
& \sum_{i \in \mathcal{N}}\left[\int_{\omega_{i}} \beta \cdot\left(w-w_{i}\right) \operatorname{Curl} \zeta d x\right] \\
& \leq\|\beta\|_{1, \infty}\|\operatorname{Curl} \zeta\|_{0, \omega}\left\|\left(w-w_{i}\right)\right\|_{0, \omega} \\
& \leq C\|\beta\|_{1, \infty}\left(\sum_{i \in \mathcal{N}} E_{(2, i)}^{2}\left(u_{h}^{N C}\right)\right)^{\frac{1}{2}}\|w\|_{\varepsilon, \omega_{i}} .
\end{aligned}
$$

Summing up the different contributions in the estimate of $\|w\|_{\varepsilon, \omega_{i}}$ and using the continuity of $a(.,$.$) yield the result.$

We have also the following result giving an estimation of expression (6):

Lemma 8 For $\zeta \in H^{1}(\Omega$ defined in (4), there exists a positive constant $C$, depending only on the minimum angle of $\mathcal{T}_{h}$ such that

$$
\varepsilon^{\frac{1}{2}}\|\operatorname{Curl} \zeta\|_{0, \Omega} \leq C\left(\sum_{i \in \mathcal{N}} E_{(2, i)}^{2}\left(u_{h}^{N C}\right)\right)^{\frac{1}{2}}
$$


Proof. Let $\zeta \in H^{1}(\Omega)$ defined in (4), we note $\zeta_{h}=\sum_{i \in \mathcal{N}} \zeta_{i} \phi$, where $\zeta_{i}=\frac{\int_{\omega_{i}} \zeta \phi_{i} d x}{\int_{\omega_{i}} \phi_{i} d x}$ for all nodes.

First, using (6) and the fact that

$$
\int_{\Omega} \varepsilon \nabla w \cdot \operatorname{Curl} \zeta d x=0
$$

Which easy to verify that:

$$
\begin{aligned}
\varepsilon\|\operatorname{Curl} \zeta\|_{0, \Omega}^{2} & =\int_{\Omega} \varepsilon\left(\nabla_{h} u_{h}^{N C}-\nabla u\right) \cdot \operatorname{Curl} \zeta d x \\
& =\int_{\Omega} \varepsilon \nabla_{h} u_{h}^{N C} \cdot \operatorname{Curl} \zeta d x,
\end{aligned}
$$

and hence:

$$
\begin{aligned}
\varepsilon\|\operatorname{Curl} \zeta\|_{0, \Omega}^{2} & =\int_{\Omega} \varepsilon \nabla_{h} u_{h}^{N C} \cdot \operatorname{Curl}\left(\zeta-\zeta_{h}\right) d x \\
& +\int_{\Omega} \varepsilon \nabla_{h} u_{h}^{N C} \cdot \operatorname{Curl} \zeta_{h} d x .
\end{aligned}
$$

On one hand, since: $\operatorname{Curl} \zeta_{h} \in H(\operatorname{div} ; \Omega), \quad \operatorname{div}\left(\operatorname{Curl} \zeta_{h}\right)=0$, and $\forall T \in \mathcal{T}_{h}, \quad\left(\operatorname{Curl} \zeta_{h}\right)_{\mid T}$ belonging the lowest Raviart-Thomas space $R T_{0}(T)=\left(P_{0}(T)\right)^{2}+\underline{x} P_{0}(T)$ and $u_{h}^{N C} \in V_{h}$, by Green formula we have

$$
\int_{\Omega} \nabla_{h} u_{h}^{N C} \cdot \operatorname{Curl} \zeta_{h} d x=0 .
$$

On the other hand, since $\left(\zeta-\zeta_{i}\right) \in V\left(\omega_{i}\right)$, using Lemma 5 , and the definition of local problem $\left(P_{2}\right)_{i}$ we have, for all $i \in \mathcal{N}$ :

$$
\begin{aligned}
& \sum_{i \in \mathcal{N}}\left[\int_{\omega_{i}} \varepsilon \nabla_{h} u_{h}^{N C} \operatorname{Curl}\left(\left(\zeta-\zeta_{i}\right) \phi_{i}\right) d x\right] \\
& =\sum_{i \in \mathcal{N}}\left[\int_{\omega_{i}} \varepsilon \nabla_{h} u_{h}^{N C} \operatorname{Curl}\left(\Pi_{i}\left(\zeta-\zeta_{i}\right) \phi_{i}\right) d x\right] \\
& =\sum_{i \in \mathcal{N}}\left[\int_{\omega_{i}} \varepsilon\left(\nabla \alpha_{i} . \nabla \Pi_{i}\left(\zeta-\zeta_{i}\right)\right) \phi_{i} d x\right]
\end{aligned}
$$

Using the last equalities, $\sum_{i \in \mathcal{N}} \phi_{i}=1$ and the equality $\|\operatorname{Curl} \zeta\|_{0, \Omega}=\|\nabla \zeta\| 0, \Omega$, we obtain

$$
\begin{aligned}
& \varepsilon\|\operatorname{Curl} \zeta\|_{0, \Omega}^{2} \\
& =\sum_{i \in \mathcal{N}} \int_{\omega_{i}} \varepsilon\left(\nabla_{h} u_{h}^{N C} \cdot \operatorname{Curl}\left(\zeta-\zeta_{h}\right)\right) \phi_{i} d x \\
& =\sum_{i \in \mathcal{N}} \int_{\omega_{i}} \varepsilon\left(\nabla_{h} u_{h}^{N C} \cdot \operatorname{Curl}\left(\Pi_{i}\left(\zeta-\zeta_{h}\right)\right)\right) \phi_{i} d x \\
& =\sum_{i \in \mathcal{N}} \int_{\omega_{i}} \varepsilon \nabla \alpha_{i} \cdot \nabla\left(\Pi_{i}\left(\zeta-\zeta_{h}\right)\right) \phi_{i} d x \\
& \leq C\left(\sum_{i \in \mathcal{N}} E_{(2, i)}^{2}\left(u_{h}^{N C}\right)\right)^{\frac{1}{2}}\left(\sum_{i \in \mathcal{N}} \int_{\omega_{i}} \varepsilon|\nabla \zeta|^{2} \phi_{i} d x\right)^{\frac{1}{2}} \\
& \leq C\left(\sum_{i \in \mathcal{N}} E_{(2, i)}^{2}\left(u_{h}^{N C}\right)\right)^{\frac{1}{2}} \varepsilon^{\frac{1}{2}}\|\operatorname{Curl} \zeta\|_{0, \Omega} .
\end{aligned}
$$

Which completes proof of the lemma.

The combination of the two lemmas 7 and 8, gives the result of the upper bound of the error of the theorem 4 .

\subsubsection{Lower bound of the error}

In this section we prove a lower bound of the error without oscillation.

Theorem 9 Let $u_{h}^{N C} \in V_{h}$, there exists a positives constants $C_{1}$ and $C_{2}$, depending on the minimum angle of the triangulation such that, for any $i \in \mathcal{N}$,

$$
E_{1, i}\left(u_{h}^{N C}\right) \leq C_{1}\left(1+\|\beta\|_{1, \infty} h_{i}\right)\left\|u-u_{h}^{N C}\right\|_{\varepsilon, w_{i}},
$$


and

$$
E_{2, i}\left(u_{h}^{N C}\right) \leq C_{2}\left\|u-u_{h}^{N C}\right\|_{\varepsilon, w_{i}}
$$

Proof. Let $i \in \mathcal{N}$. We have

$$
\begin{aligned}
E_{1, i}^{2}\left(u_{h}^{N C}\right)= & \int_{\omega_{i}}\left(\varepsilon \nabla_{h} u_{h}^{N C} \cdot \nabla \eta_{i}\right) \phi_{i} d x+\int_{\omega_{i}}\left(\beta . \nabla_{h} u_{h}^{N C} \eta_{i}\right) \phi_{i} d x \\
& -\int_{\omega_{i}} f \eta_{i} \phi_{i} d x+\frac{1}{2} \sum_{T \subset \omega_{i}} \int_{\partial T^{-}}|\beta \cdot n|\left[u_{h}^{N C}\right] \eta_{i} \phi_{i} d \sigma .
\end{aligned}
$$

Then

$$
\begin{aligned}
E_{(1, i)}^{2}\left(u_{h}\right) & =\int_{\omega_{i}}\left(\varepsilon \nabla_{h} u_{h}^{N C} \cdot \nabla \eta_{i}\right) \phi_{i} d x+\int_{\omega_{i}}\left(\beta \cdot \nabla_{h} u_{h}^{N C} \eta_{i}\right) \phi_{i} d x \\
& -\int_{\omega_{i}} f \eta_{i} \phi_{i} d x \\
& +\frac{1}{2} \sum_{T \subset \omega_{i}} \int_{\partial T^{-}}|\beta \cdot n|\left[u_{h}^{N C}\right] \eta_{i} \phi_{i} d \sigma \\
& =\int_{\omega_{i}}\left(\varepsilon\left(\nabla_{h} u_{h}^{N C}-\nabla u\right) \cdot \nabla\left(\eta_{i} \phi_{i}\right) d x\right. \\
& +\int_{\omega_{i}}\left(\beta \cdot\left(\nabla_{h} u_{h}^{N C}-\nabla u\right) \eta_{i}\right) \phi_{i} d x \\
& +\frac{1}{2} \sum_{T \subset \omega_{i}} \int_{\partial T^{-}}|\beta \cdot n|\left[u_{h}^{N C}\right] \eta_{i} \phi_{i} d \sigma \\
& =\int_{\omega_{i}}\left(\varepsilon\left(\nabla_{h} u_{h}^{N C}-\nabla u\right) \cdot \nabla\left(\eta_{i}\right) \phi_{i} d x\right. \\
& +\int_{\omega_{i}}\left(\varepsilon\left(\nabla_{h} u_{h}^{N C}-\nabla u\right) \cdot \nabla\left(\phi_{i}\right) \eta_{i} d x\right. \\
& +\int_{\omega_{i}}\left(\beta \cdot\left(\nabla_{h} u_{h}^{N C}-\nabla u\right) \eta_{i}\right) \phi_{i} d x \\
& +\frac{1}{2} \sum_{T \subset \omega_{i}} \int_{\partial T^{-}}|\beta \cdot n|\left[u_{h}^{N C}\right] \eta_{i} \phi_{i} d \sigma .
\end{aligned}
$$

Using Cauchy-Schwarz theorem imply that:

$$
\begin{aligned}
E_{(1, i)}^{2}\left(u_{h}^{N C}\right) & \leq \varepsilon^{\frac{1}{2}}\left\|\nabla u-\nabla_{h} u_{h}^{N C}\right\|_{0, \omega_{i}}\left(\varepsilon \int_{\omega_{i}}\left|\nabla \eta_{i}\right|^{2} \phi_{i} d x\right)^{\frac{1}{2}} \\
& +\varepsilon^{\frac{1}{2}}\left\|\nabla u-\nabla_{h} u_{h}^{N C}\right\|_{0, \omega_{i}} \varepsilon^{\frac{1}{2}}\left\|\eta_{i}\right\|_{0, \omega_{i}}\left\|\phi_{i}\right\|_{1, \infty, \omega_{i}} \\
& +\|\beta\|_{0, \infty}\left\|\nabla u-\nabla_{h} u_{h}^{N C}\right\|_{0, \omega_{i}}\left(\int_{\omega_{i}}\left|\eta_{i}\right|^{2} \phi_{i} d x\right)^{\frac{1}{2}} \\
& +C\left\|\left[u_{h}^{N C}\right]\right\|_{L^{2}(\partial T)}\left\|\eta_{i}\right\|_{0, \omega_{i}} .
\end{aligned}
$$

Or:

$$
\left.\varepsilon^{\frac{1}{2}}\left\|\eta_{i}\right\|_{0} \leq C h_{i} \varepsilon^{\frac{1}{2}}\left(\int_{\omega_{i}}\left(\left|\nabla \eta_{i}\right|^{2} \phi_{i} d x\right)\right)\right)^{\frac{1}{2}}
$$

Then

$$
\varepsilon^{\frac{1}{2}}\left\|\eta_{i}\right\|_{0, \omega_{i}} \leq C h_{i} E_{(1, i)}\left(u_{h}^{N C}\right)
$$

and since $\eta_{i} \in V\left(\omega_{i}\right)$, we have $\left|\phi_{i}\right|_{1, \infty, \omega_{i}} \leq \frac{C}{h_{i}}$.

And:

$$
\begin{aligned}
& \sum_{T \in \mathcal{T}_{h}} \int_{\partial T^{-}}|\beta . n|\left[u_{h}^{N C}\right] \eta_{i} \phi_{i} d \sigma \\
& \leq C\left\|\left[u_{h}^{N C}\right]\right\|\left\|_{L^{2}(\partial T)}\right\| \eta_{i} \|_{0, \omega_{i}} \\
& \leq C \varepsilon^{\frac{-1}{4}} \alpha_{T}^{\frac{1}{2}}\left\|\left[u_{h}^{N C}\right]\right\|_{L^{2}(\partial T)} E_{1, i}\left(u_{h}^{N C}\right) \\
& \leq C\left\|u-u_{h}^{N C}\right\|_{\varepsilon, w_{i}} E_{1, i}\left(u_{h}^{N C}\right) .
\end{aligned}
$$


Summing up the different contributions in the estimate of $E_{1, i}\left(u_{h}^{N C}\right)$ we get:

$$
E_{1, i}\left(u_{h}^{N C}\right) \leq C\left(1+\|\beta\|_{1, \infty} h_{i}\right)\left\|u-u_{h}^{N C}\right\|_{\varepsilon, w_{i}} .
$$

To prove the second inequality, let us remark that, using Green formula, we have :

$$
\forall \mu_{i} \in \mathcal{P}_{0}^{2}\left(\omega_{i}\right) ; \quad \int_{\omega_{i}} \varepsilon \nabla u . \operatorname{Curl}\left(\mu_{i} \phi_{i}\right) d x=0 .
$$

Then using Cauchy-Schwartz inequality we deduce

$$
\begin{aligned}
E_{(2, i)}^{2}\left(u_{h}^{N C}\right) & =\int_{\omega_{i}} \varepsilon \nabla_{h} u_{h}^{N C} \cdot \operatorname{Curl}\left(\alpha_{i} \phi_{i}\right) d x \\
& =\int_{\omega_{i}} \varepsilon\left(\nabla_{h} u_{h}^{N C}-\nabla u\right) \cdot \operatorname{Curl}\left(\alpha_{i} \phi_{i}\right) d x \\
& \leq \varepsilon^{\frac{1}{2}}\left\|\nabla u-\nabla u_{h}^{N C}\right\|_{0, w_{i}} \varepsilon^{\frac{1}{2}}\left\|\operatorname{Curl}\left(\alpha_{i} \phi_{i}\right)\right\|_{0, w_{i}} .
\end{aligned}
$$

Where

$$
\varepsilon^{\frac{1}{2}}\left\|\operatorname{Curl}\left(\alpha_{i} \phi_{i}\right)\right\|_{0, w_{i}} \leq C E_{(2, i)}\left(u_{h}^{N C}\right)
$$

Then

$$
E_{(2, i)}\left(u_{h}^{N C}\right) \leq C \varepsilon^{\frac{1}{2}}\left\|\nabla u-\nabla u_{h}^{N C}\right\|_{0, w_{i}} \leq C\left\|u-u_{h}^{N C}\right\|_{\varepsilon, w_{i}}
$$

\section{Mesh adaption procedure and Numerical results}

Example 1. We now consider, on the same domain $\Omega=] 0,1[\times] 0,1[$, the convection diffusion reaction problem:

$$
-\epsilon \Delta u+\beta . \nabla u=f
$$

with $u=0$ on $\partial \Omega$ such that $\beta=[3,2]^{t}, \epsilon=0.01$, and $f=1$

This solution, for small $\epsilon>0$, exhibits boundary layers near the top $(y=1)$ and right $(x=1)$ boundaries. The adaptive finite element method correctly refines in these layers, yielding accurate solutions with a small number of unknowns (relative to uniform refinement). The meshes and the contour maps are omitted for brevity reasons (see Fig 1 and Fig 2).

The interesting point of this problem concerns the exactness of estimators as $\epsilon$ goes to zero

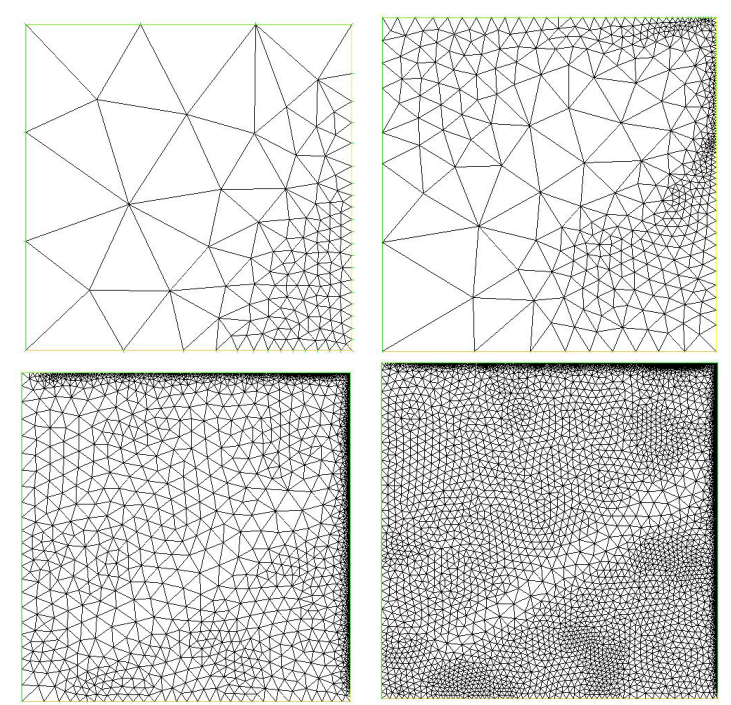

Figure 1. Adaptive mesh refinement using the error indicator. 


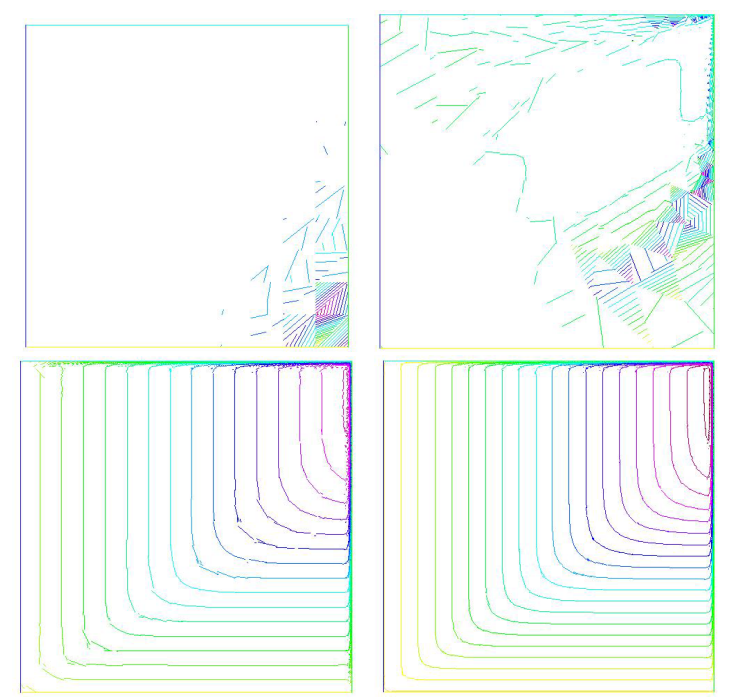

Figure 2. The isovalus of the solution .

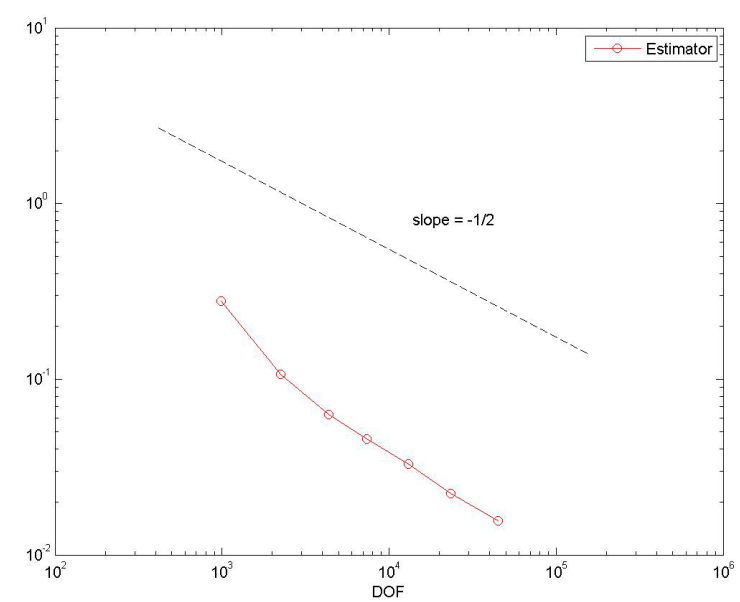

Figure 3. Decay of error indicator. The dashed line has slope of $-\frac{1}{2}$.

Example 2. We now consider, on the same domain $\Omega=] 0,1[\times] 0,1[$, the convection diffusion reaction problem:

$$
-\epsilon \Delta u+\beta . \nabla u=f .
$$

With the source term $f$ given by the exact solution,

$$
u=x y(x-1)(y-1) e^{-100(x-0.5)^{2}-100(y-0.5)^{2}}
$$

which presents sharp curvature in the vicinity of point $(0.5,0.5)$, and we perform a nonconforming finite element discretization on it. Successive iterations of adaptive mesh are represented in Figure 4. Computed and Exact solution are given in Figure 5, where the scaling of the height is the same for both pictures.

\section{Remarks.}

1. We can write a general framework regroups the conforming and nonconforming approximations of the convection diffusion problem, just write the approximate problem as:

$$
\left(P_{h}\right) \quad\left\{\begin{array}{l}
\text { Find } u_{h} \in W_{h}, \text { such that }: \forall v_{h} \in W_{h}, \\
\sum_{T \in \mathcal{T}_{h}} \int_{T}\left[\varepsilon \nabla u_{h} . \nabla v_{h}\right. \\
\left.+\beta . \nabla u_{h} v_{h}\right] d x+\frac{1}{2} \sum_{T \in \mathcal{T}_{h}} \int_{\partial T^{-}} \beta . n\left[u_{h}\right] v_{h} d \sigma \\
=\int_{\Omega} f v_{h} d x .
\end{array}\right.
$$

with $\left.W_{h}=V_{h} \cap H_{0}^{1}(\Omega)\right)$ in the conforming case and $W_{h}=V_{h}$ in the non conforming case. The same arguments can be used to derive an a posteriori error estimate on stars with similar properties.

2. The three dimensional case can be made with some regularity assumptions of the domain $\Omega$, and by adapting the used technics in the two dimensional case. 


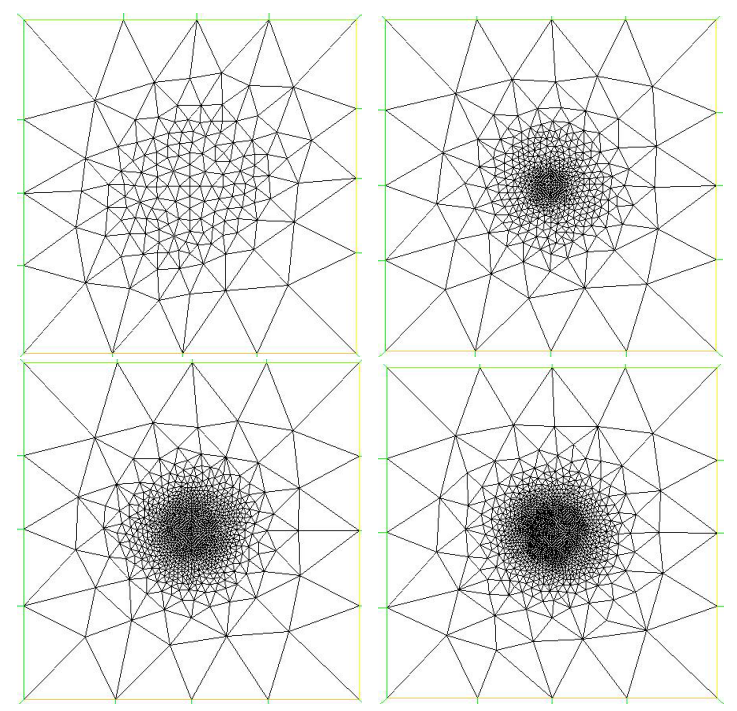

Figure 4. Adaptive mesh refinement using the error indicator.

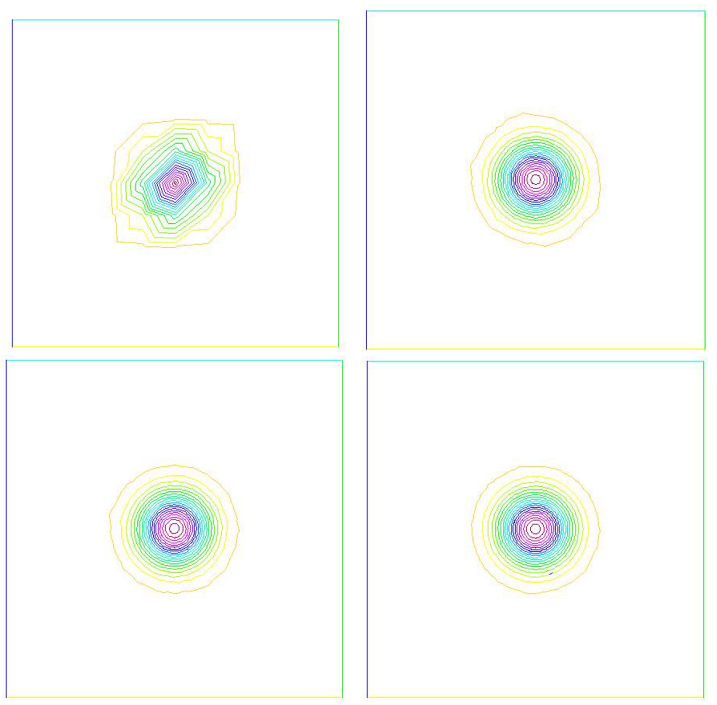

Figure 5. The isovalus of the solution .

\subsection{Conclusion}

In this work we analyzed an a posteriori error estimator for nonconforming convection diffusion problem, with the Helmholtz-Decomposition technics. These estimators are efficient and robust to respect to physical parameters of the problem.

\section{Acknowledgement}

This work is supported by the Project VOLUBILIS A.I number M.A/13/286.

\section{REFERENCES}

[1] B. Achchab, A. Agouzal, K. Bouihat and N. Debit. Star-based a posteriori error estimates for elliptic problems. (To appear)

[2] B. Achchab, A. Agouzal, A. El Fatini, A. Souissi. Robust hierarchical a posteriori error estimates for stabilized convection-diffusion problem, Numer. Math. Part. Diff. Equ. 28 (5), pp 1717-1728, 2012.

[3] B. Achchab, M. El Fatini, A. Ern, A. Souissi. A posteriori error estimates for subgrid viscosity stabilized approximations of convection-diffusion equations , Appl. Math. Lett., 22 (9), pp 1418-1424, 2009. 
[4] A. Agouzal. A posteriori error estimator for nonconforming finite element methods. Appl. Math. Lett, 7 (5), pp 1017-1033, 1994.

[5] M. Ainsworth, I. Babuska. Reliable and robust a posteriori error estimating for singularly perturbed reaction-diffusion problems. SIAM J. Numer. Anal., 36, pp 331- 353, 1999.

[6] A. Alonso. Error estimators for a mixed method. Numer. Math., 74, pp 385-395. 1996.

[7] R.E. Bank and A. Weiser. Some a posteriori error estimators for elliptic partial differential equations, Math. Comp., 44, pp 283-301. 1985.

[8] K. Bouihat. Estimations d'erreur pour les mthodes des lments finis non conformes et des volumes finis, Thse nationale de l'Université Hassan $1^{\text {er }}$ Settat, Facult des Sciences et Techniques Settat. 17 mai 2012.

[9] D. Braess and R. Verfürth. Error estimators for a the Raviart-Thomas element SIAM J. Numer. Anal., 33, pp 2431-2444. 1996.

[10] A. Brook, T. Hughes. Streamline Upwind/Petrov-Galerkin formulation for convection dominated ow with particular emphasis on the incompressible Navier-Stokes equations, Comp. meth. Appl. Mech. Engng. 32, pp 199-259, 1982.

[11] C. Carstensen, J. Hu. A unifying theory of a posteriori error control for nonconforming finite element methods. Numer. Math., 107 (3), pp 473-502, 2007.

[12] E. Dari R. Durán and C Padra. Error estimators for nonconforming finite element approximations of the Stokes problem Math. Comp., 64 (211), pp 1017-1033. 1995.

[13] V. John, G. Matthies, F. Schieweck, and L. Tobiska. A streamline-diffusion method for nonconforming finite element approximations applied to convection-diffusion problems. Comput. Methods Appl. Mech. Engrg., 166, pp 85-97, 1998.

[14] P. Knobloch and L. Tobiska. The $P_{1}^{\text {mod }}$ element: a new nonconforming finite element for convection-diffusion problems. SIAM, J. Numer. Anal., 41 (2), pp 436-456, 2003.

[15] P. Morin, R.H. Nochetto and K.G. Siebert. Local Problems on Stars: A Posteriori Error Estimators, Convergence, and Performance. Math. Comp., 72 (243), pp 1067-1097. 2003.

[16] K. W. Morton. Numerical solution of Convection-Diffusion problems. Chapman and Hall, London, 1996.

[17] R.H. Nochetto. Removing the saturation assumption in a posteriori error analysis. Insit. Lombardo. Sci. Lett. Rend. A. 127, pp 67-82. 1993.

[18] R. Temam. Navier-Stokes equations, volume 2, studies in Mathematics and its Applications. North-Holand, 1977.

[19] R. Verfürth. Robust a posteriori error estimates for stationary convection-diffusion equations. SIAM, J. Numer. Anal.,43, pp 1766-1782, 2005.

[20] R. Verfürth. A posteriori error estimators for convection-diffusion equations. Numer. Math. 80, pp 641-663, 1998.

[21] M. Vohralik. A posteriori error estimates for lowest-order-mixed finite element discretizations of convection-diffusionreaction equations. SIAM, J. Numer. Anal., 48, pp 1570-1599, 2007. 